\title{
NCI CTEP SDC Nasopharyngeal Cancer Sub-Category Terminology
}

National Cancer Institute

\section{Source}

National Cancer Institute. NCI CT EP SDC Nasopharyngeal Cancer Sub-Category

Terminology. NCI Thesaurus. Code C103076.

Nasopharyngeal Cancer is an NCl Cancer Therapeutic Evaluation Program (CTEP)

Simplified Disease Classification (SDC) category used to organize cancer-related disease

coding that harmonizes with and supports reporting based on the global standard

Medical Dictionary for Drug Regulatory Reporting (MedDRA) terminology. 